\title{
Efeito do gradiente altitudinal/latitudinal sobre espécies de aves florestais da família Furnariidae na Bacia do Rio Tibagi, Paraná, Brasil
}

\author{
Fernando de L. Fávaro; Luiz dos Anjos; Edson V. Lopes; Luciana B. Mendonça \& Graziele H. Volpato
}

Departamento de Biologia Animal e Vegetal, Universidade Estadual de Londrina. Caixa Postal 6001, 86051-990 Londrina, Paraná, Brasil. E-mail: fernandobiologo@yahoo.com.br

\begin{abstract}
Effect of altitudinal/latitudinal gradient about forest ovenbirds species (Aves: Furnariidae) in the Tibagi river basin, Paraná, Brazil. Variations in richness and abundance of forest ovenbirds (Aves: Furnariidae) were studied along an altitudinal/latitudinal gradient of the Tibagi river basin (TRB), southern Brazil. Mixed Temperate Rain Forest (1060 m a.s.l.) is the dominant forest in the southern TRB while Seasonal Semideciduous Forest (298 $\mathrm{m}$ a.s.I.) occurs in the northern. The total number of species obtained in the TRB was 14. Richness and Abundance of Furnariidae were greater in the southern (12 species, IPA = 2.01) than in the northern (five species, IPA $=0.45$ ) TRB. Similarities were also greatest in the southern and in the middle TRB. The community structure of Furnariidae also presented a positive correlation between the southern and the middle TRB (Spearman's rank correlation coefficient; $r_{s}=0.96, r_{s}=0.89, r_{s}=1.00$ ). Evolutionary relationships of some representatives of Furnariidae with southern South America and with the Andes may explain the greater presence of this family in the southern TRB. Another aspect that also may help to elucidate the results presented by Furnariidae are the resemblances between the Mixed Temperate Rain Forest of the southern TRB and others temperate forests from South America.
\end{abstract}

KEY WORDS. Altitude; latitude; mixed temperate rain forest; seasonal semideciduous forest.

RESUMO. Variações na riqueza e abundância de Furnariidae florestais foram analisadas ao longo do gradiente altitudinal/latitudinal da bacia do rio Tibagi (BRT), Paraná, Brasil. A Floresta Ombrófila Mista (1060 m a.n.m.) é a formação dominante no sul da BRT, enquanto que a Floresta Estacional Semidecidual (298 m a.n.m.), ocorre no norte. $\mathrm{O}$ número total de espécies obtidas na BRT foi 14. A riqueza e a abundância de Furnariidae foram maiores no sul $(12$ espécies, IPA $=2,01$ ) do que no norte (cinco espécies, IPA $=0,45$ ) da BRT. As similaridades também foram altas entre o sul e o centro da BRT. A estrutura da comunidade de Furnariidae também apresentou correlação positiva entre o sul e o centro da BRT (coeficiente de correlação por postos de Spearman; $r_{s}=$ $\left.0,96, r_{s}=0,89, r_{s}=1,00\right)$. As relações evolutivas de alguns representantes de Furnariidae com o sul da América do Sul e com os Andes podem explicar a maior presença desta família no sul da BRT. Um outro aspecto que também pode ajudar a explicar os resultados apresentados por Furnariidae são as semelhanças entre a Floresta Ombrófila Mista do sul da BRT e outras florestas temperadas da América do Sul.

PALAVRAS CHAVES. Altitude; floresta estacional semidecidual; floresta ombrófila mista; latitude.

Comunidades mudam à medida que ocorre variação dos fatores abióticos ao longo de um gradiente espacial, como em relação à latitude onde a riqueza de espécies tende a aumentar das latitudes maiores em direção às menores (PIANKA 1966, STEVENS 1989, GASTON 2000). Embora muitas explicações para a correlação entre latitude e riqueza de espécies (tais como história, taxas de predação e diferenças na produtividade, entre outros) também possam ser aplicadas às correlações altitudinais, raramente o efeito da mudança altitudinal foi considerado distinto daquele da mudança latitudinal (Stevens 1992). Apesar de os trabalhos pioneiros de Whittaker (Whittaker \& Niering 1965, 1975, Whittaker 1967), Terborgh (1971) e MacArthur (1972) terem fornecido a base para a realização de estudos que objetivavam elucidar a estrutura de comunidades ao longo de gradientes altitudinais, o interesse por esse tema ocorreu somente mais tarde e, em sua maioria, em regiões montanhosas (e.g. NAVARRo 1992). São poucos os estudos sobre gradiente altitudinal em bacias hidrográficas (e.g. ReYnaud 1998).

Furnariidae é uma das famílias de maior riqueza de espécies da região Neotropical, com um total de 236 espécies (REMSEN 2003). Deste total, 102 espécies ocorrem no Brasil (Comitê BRASileiro de Registros Ornitológicos 2005). As aves da família Furnariidae se destacam tanto pela homogeneidade quanto pela heterogeneidade de suas características (REMsen 2003). A 
homogeneidade é observada na coloração da plumagem (predominantemente marrom), nos sistemas sociais e na alimentação. Por outro lado, a arquitetura do ninho, a forma das estruturas, o comportamento de forrageio e a ocupação de habitats refletem a heterogeneidade dessa família.

A diversidade de habitats ocupados pelos furnarídeos é bastante ampla, podendo ocorrer desde regiões montanhosas, onde são mais comuns nas altitudes maiores (BLAKE \& LoISELlE 2000), até áreas costeiras, passando por florestas, desertos e planícies, entre outros. Tal quantidade de ambientes ocupados denota alta flexibilidade ecológica (REMSEN 2003).

Comunidades de aves florestais foram avaliadas em relação ao gradiente altitudinal da bacia do rio Tibagi (BRT), o que resultou na melhor compreensão dos padrões de distribuição das aves e, dentre estes, o da família Furnariidae que apresentou riqueza e abundância maiores na porção mais alta da BRT (Anjos 2002b). O presente estudo teve como objetivo analisar a riqueza e a abundância de aves florestais da família Furnariidae ao longo do gradiente altitudinal/latitudinal da bacia do rio Tibagi (BRT), sul do Brasil, para avaliar se o padrão apresentado por Anjos (2002b) se mantém. Paralelamente, uma análise sobre a estrutura da comunidade de Furnariidae florestais ao longo da BRT também foi desenvolvida.

\section{MATERIAL E MÉTODOS}

\section{Área de estudo}

A BRT abrange uma área de $24.712 \mathrm{~km}^{2}$ e está localizada na porção centro-leste do estado do Paraná, entre $22^{\circ} 47^{\prime} 22^{\prime \prime}$ $25^{\circ} 36^{\prime} 25^{\prime \prime} \mathrm{S}$ e $49^{\circ} 36^{\prime} 00^{\prime \prime}-51^{\circ} 27^{\prime} 36^{\prime \prime} \mathrm{W}$ (DE FRANÇA 2002), com altitudes variando entre $1060 \mathrm{~m}$ a.n.m (sul) e $298 \mathrm{~m}$ a.n.m (norte). O clima (Classificação de Köppen) varia de CfB, subtropical úmido com verão quente à moderadamente quente, no sul da BRT a Cfa, subtropical úmido com verão quente, no norte da BRT, com temperatura e pluviosidade médias de $18^{\circ} \mathrm{C}$ e 1550 $\mathrm{mm}$ e $21^{\circ} \mathrm{C}$ e $1600 \mathrm{~mm}$, respectivamente (MendonçA \& DanniOLIVEIRA 2002).

Quatro localidades de amostragem foram selecionadas : Reserva do Tarumã (RT, $25^{\circ} 18^{\prime} \mathrm{S}, 49^{\circ} 49^{\prime} \mathrm{W}, 840$ ha, $1.010 \mathrm{~m}$ a.n.m.), Floresta Nacional de Irati (FI, $25^{\circ} 24^{\prime}$ 'S, 50 $35^{\prime} \mathrm{W}, 3.495$ ha, $920 \mathrm{~m}$ a.n.m.), Parque Ecológico Klabin (PK, $24^{\circ} 17^{\prime} \mathrm{S}$, $50^{\circ} 35^{\prime} \mathrm{W}, 11.116$ ha, $815 \mathrm{~m}$ a.n.m.) e Parque Estadual Mata dos Godoy (MG, $23^{\circ} 27^{\prime} \mathrm{S}, 51^{\circ} 14^{\prime} \mathrm{W}, 656 \mathrm{ha}, 610 \mathrm{~m}$ a.n.m.).

A RT (1.010 m a.n.m.),está localizada nos municípios de Palmeira e Campo Largo. Com topografia levemente acidentada, a vegetação na RT é a Floresta Ombrófila Mista, na qual o pinheiro-do-paraná, Araucaria angustifolia (Bertol.) Kuntze (Araucariaceae) é a espécie arbórea dominante.

A FI (920 m a.n.m.) localiza-se nos municípios de Fernandes Pinheiro e Teixeira Soares e é uma unidade de conservação de uso sustentável pertencente ao Instituto Brasileiro do Meio Ambiente e dos Recursos Naturais Renováveis (IBAMA). Neste local encontra-se a maior superfície contínua de Floresta Ombrófila Mista, legalmente protegida, do estado do Paraná (1.272 ha). O restante da área é constituído por reflorestamentos de A. angustifolia, Pinus spp. e Eucalyptus spp.

O PK (815 m a.n.m.) está localizado no município de Telêmaco Borba e é de propriedade da empresa Klabin Florestal Paraná. Da área total, 7.883 ha são ocupados por remanescentes de floresta nativa, interligados por diversos cursos d'água, e o restante é ocupado por reflorestamentos de A. angustifolia, Eucalyptus spp. e Pinus spp. A floresta do PK apresenta aspectos tanto da Floresta Ombrófila Mista como da Floresta Estacional Semidecidual, podendo ser caracterizada como uma área de transição.

O MG (610 m a.n.m.) está localizado no município de Londrina e é o maior e mais bem preservado remanescente de Floresta Estacional Semidecidual do norte do Estado do Paraná. Há espécies emergentes como Aspidosperma polyneuron Muell. Arg. (Apocynaceae) e Galesia integrifolia (Spreng.) Herms. (Phytolaccaceae), esparsamente distribuídas, e a vegetação do sub-bosque é relativamente aberta.

\section{Procedimentos de campo}

Foram realizadas quatro visitas, uma por estação, em cada um dos locais, de outubro de 2003 (primavera) a julho de 2004 (inverno). O período de permanência em cada local foi de dois dias. O número de contatos de cada espécie foi estimado pelo método de amostragem por pontos de escuta (VIelliard \& Silva 1990) com algumas alterações. Em cada local foram estabelecidas duas transecções, de $1.000 \mathrm{~m}$ de extensão, paralelas (distantes $300 \mathrm{~m}$ uma da outra) e com seis pontos de amostragem cada uma (P1, P2, P3, P4, P5 e P6), distantes $200 \mathrm{~m}$ um do outro. A opção por transecções mais extensas e com um maior número de pontos de amostragens foi para cobrir uma área maior em cada local (estimada em 48 ha), enquanto que a distância de $200 \mathrm{~m}$ entre pontos foi para diminuir a possibilidade de indivíduos serem contados mais de uma vez (Bibby et al. 1993, Develey 2003). Cada transecção encontrava-se a pelo menos $100 \mathrm{~m}$ de distância da borda. A ordem de pontos empregada em todas as amostragens foi P2, P4, P6, P5, P3 e P1. Essa definição permitiu que o ponto mais interno (P6) fosse amostrado ainda na primeira metade do trabalho, quando a atividade das aves é maior. O tempo de permanência em cada ponto foi de 15 minutos e este mesmo tempo foi utilizado para o deslocamento entre os pontos. As amostragens começaram ao amanhecer no ponto inicial (P2), com o início das atividades das aves, e se encerraram cerca de três horas depois, no ponto final (P1). Desta forma, um total de 48 amostragens pontuais foram obtidas em cada localidade. A partir do número de contatos foi obtido o valor da abundância de cada espécie, o qual é expresso na forma de um índice, denominado Índice Pontual de Abundância (IPA), que é calculado dividindo-se o número total de contatos da espécie pelo número de pontos amostrados em cada local.

As categorias de forrageio foram definidas a partir de literatura (Willis 1979, Stotz et al. 1996, Sick 1997, ReMSEN 2003) e observações em campo como: solo, sub-bosque, de estrato médio, dossel, bambu e tronco. 


\section{Análise dos dados}

As similaridades (composição de espécies e número de contatos) foram comparadas entre localidades e baseadas nos índices de Sørenson e Morisita-Horn (MagurRan 1988), respectivamente. Foi testada a correlação (r, Fowler \& CoHEN 1996) entre o número de espécies e a altitude de cada localidade. O coeficiente de correlação por postos de Spearman ( $\mathrm{r}_{\mathrm{S}}$, CALLEGARI-JACQUeS 2003) foi utilizado para testar a relação das categorias de forrageio $(\mathrm{n}=$ 6) entre as localidades. Diferenças na riqueza e na abundância, das espécies e das categorias de forrageio, entre as localidades foram avaliadas por meio do teste $\mathrm{G}(\mathrm{G}=2 \times \Sigma \mathrm{O} \times \ln \mathrm{O} / \mathrm{E}$, onde: (O) freqüência observada, (E) freqüência esperada - média de $\mathrm{O}$ -, ( $($ ) somatório dos produtos $\mathrm{O} x \ln \mathrm{O} / \mathrm{E}$, (ln) logaritmo natural, o qual utiliza as mesmas tabelas do teste qui-quadrado (Fowler \& CoHen 1996). Todos os testes usaram a decisão de $\alpha=0,05$.

\section{RESULTADOS}

Um total de 14 espécies foi registrado nas quatro localidades de estudo (Tab. I), sendo 13 espécies florestais e uma, Lochmias nematura (Lichtenstein, 1823), de ambiente aquático: 12 na RT, nove na FI, nove na PK, e cinco no MG. O aumento no número de espécies foi positiva e significativamente correlacionado com o aumento da altitude $(\mathrm{r}=0,96, \mathrm{gl}=2$, $\mathrm{p}<0,05$ ). O somatório das abundâncias das espécies da família Furnariidae (Tab. II) mostrou-se significativamente diferente $(\mathrm{G}=114,72, \mathrm{gl}=3, \mathrm{p}<0,001)$ entre as localidades amostradas.
As áreas mais semelhantes na composição (índice de Sørenson) e na abundância (índice de Morisita-Horn) de espécies foram RT, FI e PK (Tab. II). Duas espécies, Clibanornis dendrocolaptoides (Pelzeln, 1859) e L. nematura foram registradas somente na RT, enquanto Cranioleuca pallida (Wied, 1831) e Automolus leucophthalmus (Wied, 1821) apareceram apenas na FI e no MG, respectivamente. Synallaxis ruficapilla (Vieillot, 1819) e Philydor rufum (Vieillot, 1818) foram registrados em todas as localidades.

Tabela II. Índices de similaridade de Sørenson e de Morisita-Horn entre os locais de amostragem, na bacia do rio Tibagi, Paraná. (RT) Reserva do Tarumã, (FI) Floresta Nacional de Irati, (PK) Parque Ecológico Klabin, (MG) Parque Estadual Mata dos Godoy.

\begin{tabular}{lcccc}
\hline & RT & FI & PK & MG \\
\hline Sørenson & & & & \\
RT & - & 0,76 & 0,86 & 0,47 \\
FI & - & - & 0,89 & 0,29 \\
PK & - & - & - & 0,43 \\
MG & - & - & - & - \\
Morisita-Horn & & & & \\
RT & - & 0,93 & 0,88 & 0,11 \\
FI & - & - & 0,91 & 0,06 \\
PK & - & - & - & 0,11 \\
MG & - & - & - & - \\
\hline
\end{tabular}

Tabela I. Abundância (IPA) e categoria de forrageio das espécies de furnarídeos nos locais de amostragem, na bacia do rio Tibagi, Paraná. (RT) Reserva do Tarumã, (FI) Floresta Nacional de Irati, (PK) Parque Ecológico Klabin, (MG) Parque Estadual Mata dos Godoy. Para a determinação das categorias de forrageio ver WiLus (1979), Stotz et al. (1996), Sick (1997) e RemSen (2003).

\begin{tabular}{|c|c|c|c|c|c|}
\hline \multirow{2}{*}{ Espécies } & \multicolumn{4}{|c|}{ Abundância } & \multirow{2}{*}{ Categoria de forrageio } \\
\hline & RT $(1.010 \mathrm{~m})$ & $\mathrm{FI}(920 \mathrm{~m})$ & PK $(815 \mathrm{~m})$ & MG $(610 \mathrm{~m})$ & \\
\hline Clibanornis dendrocolaptoides & 0,02 & - & - & - & Solo \\
\hline Leptasthenura setaria & 0,63 & 0,58 & 0,71 & - & Dossel \\
\hline Synallaxis ruficapilla & 0,08 & 0,06 & 0,25 & 0,06 & Bambu \\
\hline Synallaxis cinerascens & 0,27 & 0,17 & 0,21 & - & Sub-bosque \\
\hline Cranioleuca obsoleta & 0,13 & 0,10 & 0,17 & - & Estrato médio \\
\hline Cranioleuca pallida & - & 0,02 & - & - & Bambu \\
\hline Syndactyla rufosuperciliata & 0,13 & 0,10 & 0,04 & - & Sub-bosque \\
\hline Anabacerthia amaurotis & 0,13 & 0,02 & 0,06 & - & Estrato médio \\
\hline Philydor lichtensteini & 0,04 & - & 0,02 & 0,08 & Estrato médio \\
\hline Philydor rufum & 0,15 & 0,02 & 0,04 & 0,08 & Dossel \\
\hline Automolus leucophthalmus & - & - & - & 0,17 & Sub-bosque \\
\hline Heliobletus contaminatus & 0,33 & 0,35 & 0,13 & - & Dossel \\
\hline Xenops rutilans & 0,04 & - & - & 0,06 & Tronco \\
\hline Lochmias nematura & 0,06 & - & - & - & Solo \\
\hline Total & 2,01 & 1,42 & 1,63 & 0,45 & \\
\hline
\end{tabular}


Das 14 espécies registradas, quatro apresentaram diferença significativa em relação à abundância, apenas nas localidades em que estiveram presentes: $S$. ruficapilla $(\mathrm{G}=19,00$; $\mathrm{gl}=3$; $\mathrm{p}<0,001$ ); Anabacerthia amaurotis (Temminck, 1823), $\mathrm{G}=8,97$, $\mathrm{gl}=2, \mathrm{p}<0,02 ;$ P. rufum $(\mathrm{G}=13,09, \mathrm{gl}=3, \mathrm{p}<0,01)$ e Heliobletus contaminatus (Berlepsch, 1885), $\mathrm{G}=12,28, \mathrm{gl}=2$, $\mathrm{p}<0,01$.

Com exceção dos forrageadores de bambu ( $G=17,04$, $\mathrm{gl}=3, \mathrm{p}<0,001)$ e dos forrageadores de tronco, as demais categorias de forrageio apresentaram aumento da abundância do norte para o sul, da altitude menos elevada para a mais elevada (Tab. III). Destacaram-se nesse aspecto os forrageadores de subbosque $(\mathrm{G}=9,76, \mathrm{gl}=3, \mathrm{p}<0,05)$, os forrageadores de estrato médio $(\mathrm{G}=18,06, \mathrm{gl}=3, \mathrm{p}<0,001)$ e os forrageadores de dossel $(\mathrm{G}=111,30, \mathrm{gl}=3, \mathrm{p}<0,001)$.

Tabela III. Categorias de forrageio e suas respectivas abundâncias nos locais de amostragem, na bacia do rio Tibagi, Panraá. (RT) Reserva do Tarumã, (FI) Floresta Nacional de Irati, (PK) Parque Ecológico Klabin, (MG) Parque Estadual Mata dos Godoy.

\begin{tabular}{lcccc}
\hline \multirow{2}{*}{ Categoria de forrageio } & \multicolumn{4}{c}{ Abundância } \\
\cline { 2 - 5 } & $\mathrm{RT}$ & $\mathrm{FI}$ & $\mathrm{PK}$ & $\mathrm{MG}$ \\
\hline Solo & 0,08 & - & - & - \\
Sub-bosque & 0,40 & 0,27 & 0,25 & 0,17 \\
Estrato médio & 0,30 & 0,12 & 0,25 & 0,08 \\
Dossel & 1,11 & 0,95 & 0,88 & 0,08 \\
Bambu & 0,08 & 0,08 & 0,25 & 0,06 \\
Tronco & 0,04 & - & - & 0,06 \\
\hline
\end{tabular}

O coeficiente de correlação por postos de Spearman indicou correlações positivas quanto às categorias de forrageio de RT e FI $\left(\mathrm{r}_{\mathrm{s}}=0,96, \mathrm{n}=6, \mathrm{p}<0,02\right)$, RT e PK $\left(\mathrm{r}_{\mathrm{s}}=0,89, \mathrm{n}=6\right.$, $\mathrm{p}<0,05)$ e de FI e PK $\left(\mathrm{r}_{\mathrm{s}}=1,00, \mathrm{n}=6, \mathrm{p}<0,01\right)$; o MG não apresentou correlação com as demais localidades (MG e RT: $r_{s}=0,74$, MG e FI: $r_{s}=0,84$, MG e PK: $\left.r_{s}=0,51, n=6, p>0,05\right)$.

A maioria das categorias de forrageio apresentou correlação positiva entre o número de espécies e as altitudes: solo $(\mathrm{r}=0,66)$, sub-bosque $(\mathrm{r}=0,89)$, estrato médio $(\mathrm{r}=0,76)$, dossel $(\mathrm{r}=0,89)$, bambu $(\mathrm{r}=0,31)$, tronco $(\mathrm{r}=-0,19)$.

\section{DISCUSSÃO}

No estado do Paraná o número total de furnarídeos é de 37 espécies (Scherer-Neto \& Straube 1995), distribuídas em diferentes tipos de habitats. Desse total, 28 espécies foram registradas na BRT, onde 21 são florestais (ANJos 2002a). Oito espécies florestais presentes no levantamento de ANJos (2002a) não foram registradas no presente estudo: Leptasthenura striolata (Pelzeln, 1856), Synallaxis hypospodia (Sclater, 1874), Anabazenops fuscus (Vieillot, 1816), P. atricapillus (Wied, 1821), P. dimidiatum (Pelzeln, 1859), Cychlocolaptes leucophrus (Jardi- ne \& Selby, 1830), X. minutus (Sparrman, 1788) e Sclerurus scansor (Ménétries, 1835), sendo que este último foi observado na RT e no PK, entretanto, fora do período de amostragem.

Sobre a avifauna em relação à altitude na BRT, ANjos (2002b) obteve, em relação à Furnariidae, uma riqueza e uma biomassa maiores na RT (15 espécies, 64 g) do que no MG (13 espécies, 18,8 g). O maior número de espécies da família Furnariidae em altitudes maiores também foi observado na Costa Rica (Blake \& Loiselle 2000) onde de um total de 10 espécies, seis foram observadas acima de $1.000 \mathrm{~m}$ de altitude. Assim como no presente estudo, representantes dos gêneros Syndactyla Reichenbach, 1853 e Anabacerthia Lafresnaye, 1842 estavam presentes nas maiores altitudes, enquanto Automolus Reichenbach, 1853 e Xenops Illiger, 1811 constavam nas porções mais baixas da localidade de estudo na Costa Rica.

As diferenças na composição de espécies vegetais das florestas Ombrófila Mista e Estacional Semidecidual na BRT (TOREZAN 2002) parecem exercer forte influência sobre Furnariidae. A Floresta Ombrófila Mista, muito semelhante às florestas temperadas sulamericanas, parece fornecer habitats mais adequados aos artrópodes (principal alimento dos furnarídeos), os quais se abrigam em meio ao súber das árvores e às epífitas e conseguem sobreviver ao rigoroso inverno desta porção da BRT (ANJos 2002b). As aves da família Furnariidae são hábeis na procura por artrópodes, vasculhando diferentes tipos de substratos como súber de árvores (Remsen 2003), folhas secas (REMSEn \& PARKer III 1984, Rosemberg 1990) e epífitas (Sillett 1994). Tal cenário explicaria a maior presença de Furnariidae na Floresta Ombrófila Mista. A relação evolutiva que alguns representantes desta família têm com o sul da América do Sul e os Andes como, por exemplo Syndactyla rufosuperciliata (Lafresnaye, 1832) (Sтотz et al. 1996) e Leptasthenura setaria (Temminck, 1824) (VAURIE 1980, REMSEN 2003) pode ser uma explicação para a maior representatividade de Furnariidae na região mais alta e mais fria da BRT.

A freqüente associação entre $L$. setaria e A. angustifolia, bastante mencionada na literatura (SICK 1997, REMSEN 2003) também pôde ser constatada no presente estudo. Entretanto, explicações para essa associação inexistem, havendo somente informações referentes à restrita distribuição geográfica (acompanhando a distribuição de A. angustifolia), nidificação e comportamento. Embora Sтотz et al. (1996) afirmem que $A$. angustifolia ocorra acima de $1.000 \mathrm{~m}$ de altitude, esta espécie também é encontrada em altitudes inferiores, como é o caso do PK (ao redor de $800 \mathrm{~m}$ ). A alta abundância de $A$. angustifolia no PK deve ser a principal causa da elevada abundância de $L$. setaria nesta localidade.

Automolus leucophthalmus distribui-se desde o Nordeste até o Sul, além de porções do Centro-Oeste. O registro feito somente no MG (norte da BRT) talvez seja em razão da semelhança entre a Floresta Estacional Semidecidual e a Floresta Ombrófila Densa, a qual é o centro evolutivo de A. leucophthalmus (Sтотz et al. 1996), e a falta de um habitat adequado, com um sub-bosque denso e sombreado (REMSEN 2003), na RT, na FI e no PK. A restri- 
ta distribuição de Anabacerthia amarotis, por porções de Floresta Atlântica no Sudeste e Sul do Brasil, juntamente com proximidade da RT (Floresta Ombrófila Mista) a aquela formação vegetacional, talvez seja a razão para a maior abundância de Anabacerthia amaurotis na RT (VAURIE 1980, REMSEN 2003). A alta abundância de $S$. ruficapilla verificada no PK talvez esteja relacionada com a ocorrência de bambuzais naquela localidade. Restrição a uma vegetação rica em bambuzais é algo freqüentemente observado nesta espécie (Remsen 2003).

$\mathrm{Na}$ BRT, os representantes florestais da família Furnariidae apresentaram um padrão de riqueza de espécies semelhante ao observado por ANJos (2002b). O número de espécies, assim como a abundância, aumentou do norte para o sul da bacia, ou seja, em direção à altitude mais alta. As explicações para este padrão encontrado podem estar nas relações evolutivas de alguns representantes de Furnariidae com o sul da América do Sul e com os Andes e na semelhança entre a Floresta Ombrófila Mista da BRT e a florestas daquelas regiões.

\section{AGRADECIMENTOS}

Jonas Rodrigues de Mattos e Edson Santana da Silva auxiliaram nos trabalhos de campo. A Profa Dra Maria A. dos Santos Alves (UERJ), o Prof. Dr. Oscar A. Shibatta (UEL), a Profa Dra Sirlei T. UEL) e o Ictiólogo Mário L. Orsi (UEL) fizeram importantes sugestões que melhoraram a versão final deste trabalho. O Instituto Ambiental do Paraná concedeu autorização para os trabalhos de campo nos quatro locais de estudo. O Instituto Brasileiro do Meio Ambiente e Recursos Naturais Renováveis, a empresa Klabin Florestal Paraná e a Paróquia Nossa Senhora das Neves (Palmeira, PR) forneceram alojamento, alimentação e auxílio para a abertura das transecções nos locais. O Programa de Pós-Graduação em Ciências Biológicas da Universidade Estadual de Londrina auxiliou na aquisição de materiais de campo e de escritório. O CNPq forneceu apoio financeiro, dentro do Programa Mata Atlântica (Assessoria de Cooperação Internacional, processo 690146/01-9). O segundo autor recebe Bolsa Produtividade em Pesquisa do CNPq (processo 350054/95-9).

\section{REFERÊNCIAS}

Anjos, L. Dos. 2002a. A avifauna da bacia do rio Tibagi, p. 271290. In: M.E. Medri; E. Bianchini; O.A. Shibatta \& J.A. Pimenta (Eds). A bacia do rio Tibagi. Londrina, Edição dos Editores, $595 p$.

AnJos, L. Dos. 2002b. Forest bird communities in the Tibagi river hydrographic basin, southern Brazil. Ecotropica, Bonn, 8: 67-79.

Bibby, C.J.; N.D. Burgess \& D.A. Hill. 1993. Bird Census Techniques. London, Academic Press, 257p.

Blake, J.G. \& B.A. Loiselle. 2000. Diversity of birds along an elevational gradient in the Cordillera Central, Costa Rica. The Auk, Missoula, 117: 663-686.
Callegari-Jacques, S.M. 2003. Bioestatística: princípios e aplicações. Porto Alegre, Artmed, 255p.

Comitê Brasileiro de Registros Ornitológicos. 2005. Listas das aves do Brasil. Versão 22/III/2005. Disponível na World Wide Web em: http://www.ib.usp.br/cbro. [Acesso em 12/ $\mathrm{XI} / 2005]$.

De FrançA, V. 2002. O rio Tibagi no contexto hidrogeográfico paranaense, p. 45-61 In: M.E. Medri; E. Bianchini; O.A. ShibatTA \& J.A. Pimenta (Eds). A bacia do rio Tibagi. Londrina, Edição dos Autores, 595p.

Develey, P.F. 2003. Métodos para estudos com aves, p. 153-168. In: L. Cullen Jr.; R. Rudran \& C. Valladares-Padua. (Eds). Métodos de estudos em biologia da conservação e manejo da vida silvestre. Curitiba, Editora da Universidade Federal do Paraná, 667p.

Fowler, J. \& L. CoHen. 1996. Statistics for ornithologists. Thetford, BTO Guide 22, 150p.

Gaston, K.J. 2000. Global patterns in biodiversity. Nature, London, 405: 220-227.

Macarthur, R.H. 1972. Geographical ecology: patterns in the distribution of species. New York, Harper \& Row, 269p.

Magurran, A.E. 1988. Ecological diversity and its measurement. Princeton, Princeton University Press, 179p.

MendonçA, F. DE A. \& I.M. Danni-Oliveira. 2002. Dinâmica atmosférica e tipos climáticos predominantes da bacia do rio Tibagi, p. 63-66. In: M.E. Medri; E. Bianchini; O.A. Shibatta \& J.A. Pimenta (Eds). A bacia do rio Tibagi. Londrina, Edição dos Autores, 595p.

Navarro, A.G. 1992. Altitudinal distribution of birds in the Sierra Madre del Sur, Guerrero, Mexico. Condor, Lawrence, 94: 29-39.

PiANKA, E.R. 1966. Latitudinal gradients in species diversity: a review of the concepts. American Naturalist, Chicago, 100: 33-46.

Remsen, J.V. 2003. Family Furnariidae (Ovenbirds), p. 162-357. In: J. Del Hoyo; A. Elliott \& D.A. Christie. (Eds). Handbook of the Birds of the World. Barcelona, Lynx Editions, vol. 8, $845 p$.

Remsen, J.V. \& T.A. PARKer III. 1984. Arboreal dead-leaf-searching birds of the Neotropics. Condor, Lawrence, 86: 36-41.

Reynaud, P.A. 1998. Changes in understory avifauna along the Synnamary river (French Guyana, South America). Ornitologia Neotropical, Quebec, 9: 51-70.

Rosenberg, K.V. 1990. Dead-leaf foraging specialization in tropical forest birds: measuring resource availability and use, p. 360-368. In: M.L. Morrison; C.J. Ralph; J. Verner \& J.R. JEHL JR. (Eds) Avian foraging: theory, methodology, and applications. Lawrence, Cooper Ornithological Society, Studies in Avian Biology, 13515p.

Scherer-Neto, P. \& F. C. Straube. 1995. Aves do Paraná: história, lista anotada e bibliografia. Ed dos autores, Curitiba, $79 \mathrm{p}$.

SICK, H. 1997. Ornitologia brasileira. Rio de Janeiro, Nova Fron- 
teira, 912p.

Sillett, T.S. 1994. Foraging ecology of epiphyte-searching insectivorous birds in Costa Rica. Condor, Lawrence, 96: 863-877.

STEvens, G.C. 1989. The latitudinal gradient in geographical range: how so many species coexist in the tropics. American Naturalist, Chicago, 133: 240-256.

Stevens, G.C. 1992. The elevational gradient in altitudinal range: an extension of Rapoport's latitudinal rule to altitude. American Naturalist, Chicago, 140: 893-911.

Stotz, D.F.; F.J.W. FitzPatrick; T.A. Parker III \& D.K. Moskovitz. 1996. Neotropical birds. Ecology and conservation. Chicago, University of Chicago Press, 481p.

Terborgh, J. 1971. Distribution on environmental gradients: theory and a preliminary interpretation of distributional patterns in the avifauna of the Cordillera Vilcabamba, Peru. Ecology, Durham, 52: 23-40.

ToreZAn, J.M. D. 2002. Nota sobre a vegetação da bacia do rio Tibagi, p. 103-107. In: M.E. Medri; E. Bianchini; O.A. ShibattA \& J.A. Pimenta (Eds). A bacia do rio Tibagi. Londrina, Edição dos Autores, 595p.

Recebido em 06.VI.2005; aceito em 03.III.2006.
VAURIE, C. 1980. Taxonomy and geographical distribution of the Furnariidae (Aves, Passeriformes). New York, American Museum of Natural History, vol. 166, 357p.

Vielliard, J.E.M. \& W.R. Silva. 1990. Nova metodologia de levantamento quantitativo da avifauna e primeiros resultados no interior do Estado de São Paulo, Brasil. In: Anais do IV Encontro Nacional dos Anilhadores de Aves, Recife, p. 117-151.

WhitTAKER, R.H. 1967. Gradients analysis of vegetation. Biological Reviews of the Cambridge Philosophical Society, Cambridge, 42: 207-264.

WhitTAKer, R.H. \& W.A. Niering. 1965. Vegetation of the Santa Catalina Mountains, Arizona. II. A gradient analysis of the south slope. Ecology, Durham, 46: 429-452.

Whittaker, R.H. \& W.A. Niering. 1975. Vegetation of the Santa Catalina Mountains, Arizona. V. Biomass, production and diversity along the elevation gradient. Ecology, Durham, 56: 771-790.

WilLIs, E.O. 1979. The composition of avian communities in remanescent woodlots in southern Brazil. Papéis Avulsos de Zoologia, São Paulo, 33: 1-25. 